\author{
Marquette University \\ e-Publications@Marquette
}

School of Dentistry Faculty Research and

Publications

Dentistry, School of

$4-2018$

\title{
Trends in Self-Reported Oral Health of US Adults: National Health and Nutrition Examination Survey 1999-2014
}

\author{
Kar Yan Li \\ University of Hong Kong \\ Christopher Okunseri \\ Marquette University, christopher.okunseri@marquette.edu \\ Colman McGrath \\ University of Hong Kong \\ May C.M. Wong \\ University of Hong Kong
}

Follow this and additional works at: https://epublications.marquette.edu/dentistry_fac

Part of the Dentistry Commons

\section{Recommended Citation}

Li, Kar Yan; Okunseri, Christopher; McGrath, Colman; and Wong, May C.M., "Trends in Self-Reported Oral Health of US Adults: National Health and Nutrition Examination Survey 1999-2014" (2018). School of Dentistry Faculty Research and Publications. 286.

https://epublications.marquette.edu/dentistry_fac/286 
Marquette University

e-Publications@Marquette

\section{Dentistry Faculty Research and Publications/School of Dentistry}

This paper is NOT THE PUBLISHED VERSION; but the author's final, peer-reviewed manuscript. The published version may be accessed by following the link in th citation below.

Community Dentistry and Oral Epidemiology, Vol. 46, No. 2 (April 2018): 203-211. DOI. This article is (C) Wiley and permission has been granted for this version to appear in e-Publications@Marquette. Wiley does not grant permission for this article to be further copied/distributed or hosted elsewhere without the express permission from Wiley.

\section{Trends in Self-Reported Oral Health Of US Adults: National Health And Nutrition Examination Survey 1999-2014}

Kar Yan Li

Faculty of Dentistry, The University of Hong Kong, Hong Kong, China

Christopher E. Okunseri

Department of Clinical Services, School of Dentistry, Marquette University Milwaukee, Milwaukee, WI, Colman McGrath

Dental Public Health, Faculty of Dentistry, The University of Hong Kong, Hong Kong, China

May C.M. Wong

Dental Public Health, Faculty of Dentistry, The University of Hong Kong, Hong Kong, China

\section{Abstract \\ Objective}

Single-item self-reported oral health $(\mathrm{SROH})$ is a convenient and reliable measure for the assessment of population-based oral health. However, little is known about trends and its associations among US 
adults. This study investigated trends in $\mathrm{SROH}$ (aged 20+ years) and the associated factors among adults living in the United States.

\section{Methods}

Self-reported oral health data for 41621 adults aged 20+ years from the National Health and Nutrition Examination Survey (NHANES) 1999 to 2014 were analysed. Survey-weighted descriptive statistics were computed to provide nationally representative estimates. Multivariable logistic regression was performed separately for each survey period with $\mathrm{SROH}$ as the primary outcome. Independent variables included were age, gender, race/ethnicity, education level and family poverty income ratio or PIR. Pooled survey-weighted multivariable logistic regression was also performed to consider possible time-changing effects.

\section{Results}

The survey-weighted proportions of "excellent or very good" in SROH increased from $27 \%$ in 1999 $2000(n=4873)$ to $38 \%$ in 2013-2014 ( $n=5765)$. Separate multivariable logistic analyses for each survey period suggested that females, Whites (vs Mexican and Black Americans) as well as respondents from high family PIR had higher odds of reporting their oral health as "excellent or very good" $(P<.05)$. The pooled multivariable logistic model confirmed results in the separate logistic regression, and respondents in the more recent survey periods had higher probabilities of reporting "excellent or very good" oral health. Respondents aged 50-59 years were found to have relatively lower probabilities of reporting "excellent or very good" oral health, while people aged 20-29 years had higher probabilities than those aged 30-39 years. Compared to respondents with lower education, those with higher education were more likely to report their oral health as excellent or very good.

\section{Conclusions}

Self-reported oral health improved from 1999 to 2014. In general, respondents who were young, female, White, had higher education or higher income or were surveyed in more recent years reported excellent or very good oral health.

\section{INTRODUCTION}

Large-scale epidemiological surveys are crucial to developing and monitoring national oral health objectives and for informing oral health policies. A balanced dental healthcare system is essential to providing and maintaining adequate access to appropriate dental care services that can bring about changes in the oral health status of an individual or a population. ${ }^{1}$ Globally, changes in oral health status for individuals or population groups are somewhat difficult to measure qualitatively or quantitatively. ${ }^{1-5}$ However, prior studies have documented some of the advantages and disadvantages of validated single-item and multi-item scales in medicine and dentistry. ${ }^{1-5}$ In addition, clinical oral health assessments are not always feasible in large epidemiological surveys due to logistics and costs. 6 To this end, the use of single-item scales of self-reported oral health ( $\mathrm{SROH}$ ) has been advocated by researchers to provide insights into the oral health of populations. ${ }^{6,7}$

The use of a single-item/global-item measures of SROH has received a lot of attention from psychosocial, gerontological and health services researchers, ${ }^{6-9}$ in part because of the brevity and the offer into individual's own subjective and objective assessment of their oral health based on their own concept or definition of it. ${ }^{4,8,9} \mathrm{SROH}$ is a cost-effective and a valid measure of oral health, ${ }^{10-14}$ with 
accepted reliability in test-retest analyses, ${ }^{15}$ and has been reported to show strong associations with other subjective oral health measures, such as oral health-related quality of life assessments. ${ }^{16}$ In addition, $\mathrm{SROH}$ has been reported to be associated with clinical oral health status such as tooth loss, dental caries experience, periodontal health status and other oral diseases and conditions. ${ }^{9,}{ }^{17,}{ }^{18}$ For example, Thompson's et al $^{9}$ reported that dental caries and tooth loss showed consistent statistically significant gradients across single-item scales with respondents reporting "excellent" having lower scores based on their analyses from national surveys conducted in New Zealand and Australia. However, little is known from large epidemiological surveys about trends in SROH over time and whether ratings for oral health improve over time among adults in the United States.

Several studies have reported on the association between SROH and socioeconomic, demographic and psychosocial variables. ${ }^{19-23}$ For example, Locker's study analysed data from the Canadian Community Health Survey of 2003 and reported that psychosocial factors in part explain the socioeconomic disparities in self-perceived oral health after controlling for tooth loss and denture wearing. ${ }^{19}$ Matthias reported on the relationship between self-rated oral health and a comprehensive set of clinical factors, sociodemographic, physical and mental health measures in an elderly urban population. ${ }^{20}$ The data revealed that "worry about teeth" and the "appearance of teeth," race/ethnicity and education were associated with self-rating of oral health. ${ }^{20}$ In addition, SROH may be influenced by cultural and competing priorities at one time or another ${ }^{21,22}$ and may vary with changing attitudes towards oral health over time. ${ }^{23}$ Furthermore, studies have reported on the relationship between socioeconomic trends in missing teeth, edentulism and SROH. ${ }^{24-28}$ While many of these studies on SROH have concentrated on older adult populations from developed countries, a gap still exists in our knowledge regarding factors that influence $\mathrm{SROH}$ over time and the consistency of these influences in the United States.

The purpose of this study was to examine trends in single-item SROH over time (a 15-year period) and their association with sociodemographic factors among adults in the United States. We hypothesized that $\mathrm{SROH}$ across socioeconomic gradient will still persist in adult populations in the United States.

\section{METHODS}

Data from the National Health and Nutrition Examination Survey (NHANES) from 1999 to 2014 were analysed (eight 2-year survey periods: 1999-2000, 2001-2002, 2003-2004, 2005-2006, 2007-2008, 2009-2010, 2011-2012 and 2013-2014). NHANES is a cross-sectional survey based on a stratified, multistage probability design, to monitor the health and nutritional status of a large age range of civilian noninstitutionalized population groups in the United States. The NHANES questionnaires are administered to participants both at home and in NHANES Mobile Examination Centers, and the data are publicly available for download. The National Center for Health Statistics (NCHS) provides extensive documentation of NHANES survey, examination and laboratory procedures on its website. ${ }^{29}$ Some subgroup populations, such as non-Hispanic Blacks and Mexican-Americans and persons aged 80 years or older, were oversampled to achieve more precise estimates for these groups. ${ }^{30}$ In this study, we only included individuals aged 20 or above with self-reported oral health information. In survey period 2009-2010, NHANES participants aged 20-29 years were not the target group in the oral health questionnaire; therefore, their self-reported oral health status was not included. 
For consistency with published studies from other national databases, self-reported oral health status (SROH) was categorized into 4 levels (excellent or very good; good; fair; poor). Age was stratified into 10-year intervals (20-29; 30-39; 40-49; 50-59; 60-69; 70-79; 80+). Race/ethnicity was described as White; Black; Mexican; Others. Educational level was separated into 4 levels ( $\leq$ Grade 12/Grade 12 with no diploma; high school graduate/General Educational Development (GED) or equivalent; some college or Associate (AA) degree; college graduate or above). Family income was determined based on an index-family poverty income ratio (PIR), so that the index could be comparable over the years. PIR was calculated by dividing family income by the poverty guidelines issued by the Department of Health and Human Services (DHHS) for each year, which were used as poverty measures for determining financial eligibility for certain federal programmes, ${ }^{31}$ specific to family size, as well as the appropriate year. Ratios below $100 \%$ were adopted as a simplified measure to indicate that family income was below the poverty level, while a ratio of $100 \%$ or greater implied family income at or above the poverty level. PIR was divided into 5 levels (<100\%; 100\%-199\%; 200\%-299\%; 300\%-399\%; 2400\%) in this study.

\subsection{Statistical analysis}

Summary and inferential statistics were performed for each survey and then pooled together. All data were analysed using SAS On Demand for Academics Version 3.4 (Enterprise Edition; SAS Institute Inc., Cary, NC, USA) software. The SURVEYFREQ and SURVEYLOGISTIC procedures in SAS were used to analyse the data incorporating Mobile Examination Center 2-year sample weights (WTMEC2YR), primary sampling units and strata which were supplied by NHANES in the data to account for NHANES' complex survey design (including over-sampling), survey nonresponse and poststratification.

Survey-weighted descriptive statistics were computed to provide nationally representative estimates. Rao-Scott chi-square test with Bonferroni was used to adjust for multiple comparisons performed to investigate the association between prevalence/severity of $\mathrm{SROH}$ and survey period. ${ }^{32}$ Survey-weighted multivariable binary logistic regressions were performed separately for each survey period to evaluate the associations of the prevalence of "excellent or very good" SROH and 5 categorical sociodemographic factors: age, gender, race/ethnicity, education level and PIR over time.

For the pooled surveys, to detect the possible time-changing effects across the surveys, surveyweighted multivariable binary logistic regression for the prevalence of "excellent or very good" SROH was conducted. This was to investigate associations with the above 5 sociodemographic factors, survey period effect and their possible interactions with survey period (period $x$ age, period $\times$ gender, period $x$ race/ethnicity, period $x$ education level and period $\times$ PIR). The interaction effects were added using a forward selection method, and predicted probabilities were estimated by odds ratios in the final model. Likelihood ratio test, Score test and Wald test were used to check the goodness-of-fit of the model. A two-sided 0.05 significance level was used for all analyses.

\section{RESULTS}

A total of 41621 individuals were included in this study, and approximately 4000-6000 individuals were included for each survey period. Figure 1 shows that the survey-weighted proportions of "excellent or very good," "good," "fair" and "poor" in SROH changed from 27\%, 38\%, 23\% and 12\% in 1999-2000 (n $=4873$ ) to $38 \%, 34 \%, 20 \%$ and $8 \%$ in $2013-2014$ ( $n=5765)$ over the years (Rao-Scott chi-square test: $P$ $<.001)$. The Bonferroni adjusted multiple comparisons showed that the proportion patterns were 
significantly different from each other in the earlier periods but becoming nonsignificant in the more recent survey periods (for example 2009-2014).

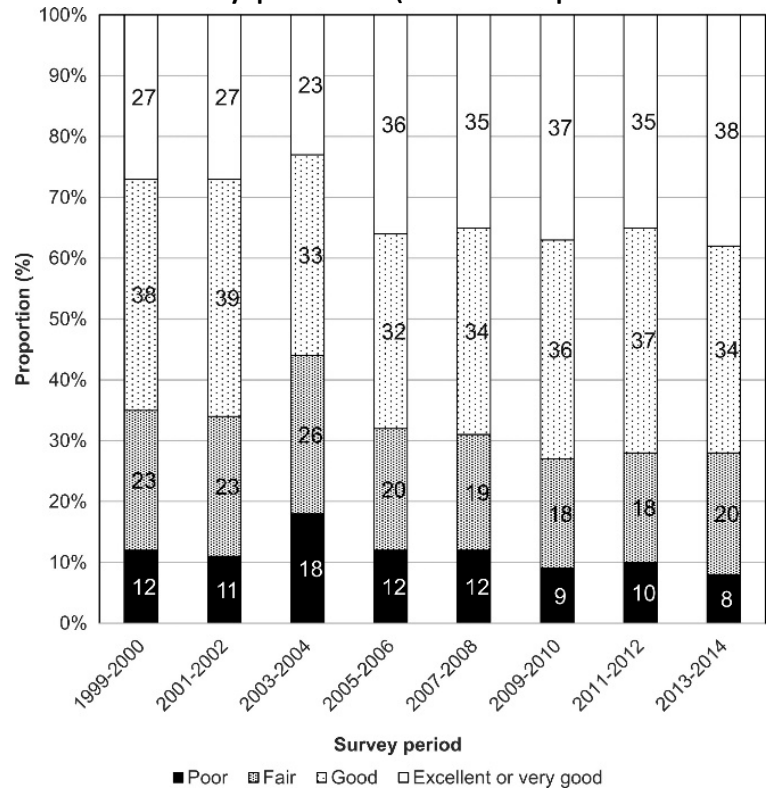

Figure 1 Survey-weighted self-reported oral health status of US adults aged 20+ years in 1999-2014

Separate survey-weighted multivariable binary logistic analyses of reporting "excellent or very good" SROH in 1999-2006 are shown in Table 1 and 2007-2014 in Table 2. In both tables, age, gender, race/ethnicity, educational level and family income (PIR) were significant factors (all $P<.05)$, but the significant patterns seen in age were not consistent over the years. Generally, compared with the age group of 30-39 years, those aged 50-59 were found to have lower odds (Odd ratio [OR] range: 0.570.90) and age group 20-29 had higher odds (OR range: 1.10-1.39) of reporting their oral health as "excellent or very good". Females had significantly higher odds of reporting their oral health as "excellent or very good" (OR range: 1.17-1.41, all $\mathrm{P}<.01$ ). 
Table 1. Multivariable analyses of factors associated with self-reported oral health (excellent or very good) of US adults: $1999-2006$

\begin{tabular}{|c|c|c|c|c|c|c|c|c|c|c|c|c|}
\hline Surveys & $\begin{array}{l}1999- \\
2000\end{array}$ & & & $\begin{array}{l}2001- \\
2002 \\
\end{array}$ & & & $\begin{array}{l}2003- \\
2004 \\
\end{array}$ & & & $\begin{array}{l}2005- \\
2006 \\
\end{array}$ & & \\
\hline Variables & OR & $(95 \% \mathrm{Cl})$ & $P$-value & OR & $(95 \% \mathrm{Cl})$ & $P$-value & OR & $(95 \% \mathrm{Cl})$ & $P$-value & OR & $(95 \% \mathrm{Cl})$ & $P$-value \\
\hline Age & & & $.013 \underline{a}$ & & & $<.001 \underline{a}$ & & & $.007 \underline{a}$ & & & $<.001 \underline{a}$ \\
\hline $20-29$ & 1.39 & $(0.92,2.08)$ & .115 & 1.24 & $(0.85,1.81)$ & .256 & 1.33 & $(1.00,1.75)$ & $.047 \underline{a}$ & 1.10 & $(0.83,1.48)$ & .502 \\
\hline $30-39$ & 1 & - & - & 1 & - & - & 1 & - & - & 1 & - & - \\
\hline $40-49$ & 1.01 & $(0.71,1.43)$ & .947 & 0.89 & $(0.71,1.12)$ & .308 & 1.06 & $(0.82,1.38)$ & .657 & 0.90 & $(0.68,1.19)$ & .444 \\
\hline $50-59$ & 0.90 & $(0.64,1.26)$ & .525 & 0.68 & $(0.52,0.90)$ & $.006 \underline{a}$ & 0.89 & $(0.64,1.23)$ & .467 & 0.67 & $(0.51,0.86)$ & $.002 \underline{\underline{a}}$ \\
\hline $60-69$ & 0.88 & $(0.58,1.32)$ & .525 & 0.96 & $(0.75,1.23)$ & .751 & 0.86 & $(0.61,1.22)$ & .407 & 1.07 & $(0.78,1.46)$ & .696 \\
\hline 70-79 & 1.08 & $(0.70,1.66)$ & .721 & 0.92 & $(0.67,1.26)$ & .606 & 1.18 & $(0.83,1.68)$ & .364 & 1.13 & $(0.78,1.63)$ & .512 \\
\hline $80+$ & 1.41 & $(0.77,2.57)$ & .269 & 1.07 & $(0.65,1.76)$ & .789 & 1.59 & $(1.16,2.18)$ & $.004 \underline{\underline{a}}$ & 0.98 & $(0.74,1.29)$ & .871 \\
\hline \multicolumn{13}{|l|}{ Gender } \\
\hline Female & 1.26 & $(1.07,1.50)$ & $.006 \underline{a}$ & 1.40 & $(1.15,1.71)$ & $.001 \underline{a}$ & 1.37 & $(1.15,1.63)$ & $<.001 \underline{a}$ & 1.41 & $(1.26,1.57)$ & $<.001 \underline{a}$ \\
\hline Male & 1 & - & - & 1 & - & - & 1 & - & - & 1 & - & - \\
\hline Race/Ethnicity & & & $.002 \underline{a}$ & & & $<.001 \underline{a}$ & & & $<.001 \underline{a}$ & & & $<.001 \underline{a}$ \\
\hline Mexican American & 0.52 & $(0.37,0.74)$ & $<.001 \underline{a}$ & 0.45 & $(0.36,0.56)$ & $<.001 \underline{a}$ & 0.38 & $(0.25,0.58)$ & $<.001 \underline{a}$ & 0.62 & $(0.50,0.76)$ & $<.001 \underline{a}$ \\
\hline Non-Hispanic Black & 0.89 & $(0.66,1.21)$ & .473 & 0.66 & $(0.55,0.80)$ & $<.001 \underline{a}$ & 0.59 & $(0.45,0.77)$ & $<.001 \underline{a}$ & 0.66 & $(0.55,0.78)$ & $<.001 \underline{a}$ \\
\hline Non-Hispanic White & 1 & - & - & 1 & - & - & 1 & - & - & 1 & - & - \\
\hline Others & 0.70 & $(0.48,1.03)$ & .068 & 0.68 & $(0.44,1.06)$ & .088 & 0.45 & $(0.34,0.59)$ & $<.001 \underline{a}$ & 0.76 & $(0.56,1.03)$ & .075 \\
\hline Education level & & & $<.001 \underline{\underline{a}}$ & & & $<.001 \underline{\underline{a}}$ & & & $<.001 \underline{\underline{a}}$ & & & $<.001 \underline{\underline{a}}$ \\
\hline $\begin{array}{l}\leq \text { Grade } 12 / \text { Grade } 12 \text { with } \\
\text { no diploma }\end{array}$ & 0.68 & $(0.49,0.94)$ & $.020 \underline{a}$ & 0.67 & $(0.47,0.94)$ & $.022 \underline{a}$ & 0.84 & $(0.62,1.13)$ & .247 & 0.64 & $(0.48,0.84)$ & $.001 \underline{a}$ \\
\hline $\begin{array}{l}\text { High School } \\
\text { graduate/GED/equivalent }\end{array}$ & 0.76 & $(0.60,0.96)$ & $.022 \underline{a}$ & 0.70 & $(0.51,0.95)$ & $.021 \underline{a}$ & 0.65 & $(0.47,0.89)$ & $.007 \underline{a}$ & 0.73 & $(0.60,0.88)$ & $.001 \underline{a}$ \\
\hline $\begin{array}{l}\text { Some college or associate } \\
\text { degree }\end{array}$ & 1 & - & - & 1 & - & - & 1 & - & - & 1 & - & - \\
\hline
\end{tabular}




\begin{tabular}{|c|c|c|c|c|c|c|c|c|c|c|c|c|}
\hline College graduate or above & 1.40 & $(1.09,1.79)$ & $.009 \underline{a}$ & 1.44 & $(1.08,1.92)$ & $.012 \underline{a}$ & 2.25 & $(1.80,2.81)$ & $<.001 \underline{a}$ & 1.81 & $(1.61,2.03)$ & $<.001 \underline{\mathrm{a}}$ \\
\hline Poverty income ratio & & & $<.001 \underline{a}$ & & & $<.001 \underline{\underline{a}}$ & & & $<.001 \underline{a}$ & & & $<.001 \underline{a}$ \\
\hline$<100 \%$ & 0.51 & $(0.39,0.66)$ & $<.001 \underline{a}$ & 0.45 & $(0.33,0.61)$ & $<.001 \underline{a}$ & 0.44 & $(0.33,0.59)$ & $<.001 \underline{a}$ & 0.48 & $(0.37,0.62)$ & $<.001 \underline{a}$ \\
\hline $100 \%-199 \%$ & 0.74 & $(0.50,1.09)$ & . 123 & 0.36 & $(0.29,0.45)$ & $<.001 \underline{a}$ & 0.50 & $(0.40,0.61)$ & $<.001 \underline{a}$ & 0.60 & $(0.48,0.75)$ & $<.001 \underline{a}$ \\
\hline $200 \%-299 \%$ & 0.46 & $(0.33,0.65)$ & $<.001 \underline{a}$ & 0.65 & $(0.52,0.82)$ & $<.001 \underline{a}$ & 0.56 & $(0.46,0.69)$ & $<.001 \underline{\underline{a}}$ & 0.59 & $(0.48,0.72)$ & $<.001 \underline{a}$ \\
\hline $300 \%-399 \%$ & 0.77 & $(0.52,1.14)$ & .188 & 0.70 & $(0.53,0.94)$ & $.018 \underline{a}$ & 0.69 & $(0.54,0.87)$ & $.002 \underline{a}$ & 0.72 & $(0.55,0.94)$ & $.015 \underline{a}$ \\
\hline$\geq 400 \%$ & 1 & - & - & 1 & - & - & 1 & - & - & 1 & - & - \\
\hline
\end{tabular}

OR, odds ratio; $95 \% \mathrm{Cl}, 95 \%$ confidence interval.

a Significant, $\mathrm{P}$-value $<.05$. 
Table 2. Multivariable analyses of factors associated with self-reported oral health (excellent or very good) of US adults: $2007-2014$

\begin{tabular}{|c|c|c|c|c|c|c|c|c|c|c|c|c|}
\hline Surveys & $\begin{array}{l}2007- \\
2008\end{array}$ & & & $\begin{array}{l}\text { 2009- } \\
\text { 2010a }\end{array}$ & & & $\begin{array}{l}2011- \\
2012\end{array}$ & & & $\begin{array}{l}2013- \\
2014\end{array}$ & & \\
\hline Variables & OR & $\begin{array}{l}(95 \% \\
\mathrm{CI})\end{array}$ & $P$-value & OR & $\begin{array}{l}(95 \% \\
\mathrm{Cl})\end{array}$ & $P$-value & OR & $(95 \% \mathrm{Cl})$ & $P$-value & OR & $95 \% \mathrm{Cl}$ & $P$-value \\
\hline Age & & & $<.001 \underline{b}$ & & & $<.001 \underline{b}$ & & & $<.001 \underline{b}$ & & & $.011 \underline{b}$ \\
\hline $20-29$ & 1.35 & $\begin{array}{l}(1.08, \\
1.69)\end{array}$ & $.009 \underline{b}$ & - & - & - & 1.23 & $\begin{array}{l}\text { (0.95, } \\
1.59)\end{array}$ & .110 & 1.34 & $\begin{array}{l}(1.04, \\
1.72)\end{array}$ & $.022 \underline{b}$ \\
\hline $30-39$ & 1 & - & - & 1 & - & - & 1 & - & - & 1 & - & - \\
\hline $40-49$ & 0.65 & $\begin{array}{l}0.57, \\
0.74)\end{array}$ & $<.001 \underline{b}$ & 0.75 & $\begin{array}{l}(0.55, \\
1.01)\end{array}$ & .056 & 0.90 & $\begin{array}{l}(0.60, \\
1.35)\end{array}$ & .606 & 1.17 & $\begin{array}{l}(0.99, \\
1.38)\end{array}$ & .069 \\
\hline $50-59$ & 0.57 & $\begin{array}{l}0.45, \\
0.72)\end{array}$ & $<.001 \underline{b}$ & 0.68 & $\begin{array}{l}(0.47, \\
1.00)\end{array}$ & $.050 \underline{b}$ & 0.66 & $\begin{array}{l}0.50, \\
0.86)\end{array}$ & $.003 \underline{b}$ & 0.89 & $\begin{array}{l}(0.70, \\
1.14)\end{array}$ & .352 \\
\hline $60-69$ & 1.00 & $\begin{array}{l}(0.78, \\
1.28)\end{array}$ & .996 & 1.14 & $\begin{array}{l}0.83, \\
1.55) \\
\end{array}$ & .419 & 0.89 & $\begin{array}{l}0.70, \\
1.14) \\
\end{array}$ & .358 & 1.29 & $\begin{array}{l}0.79, \\
2.12) \\
\end{array}$ & .304 \\
\hline $70-79$ & 0.86 & $\begin{array}{l}0.67, \\
1.11)\end{array}$ & .247 & 1.06 & $\begin{array}{l}(0.84, \\
1.33) \\
\end{array}$ & .655 & 1.46 & $\begin{array}{l}(0.98, \\
2.18) \\
\end{array}$ & .063 & 1.44 & $\begin{array}{l}(1.04, \\
2.00) \\
\end{array}$ & $.029 \underline{b}$ \\
\hline $80+$ & 0.81 & $\begin{array}{l}0.56, \\
1.16)\end{array}$ & .245 & 0.91 & $\begin{array}{l}0.66, \\
1.25)\end{array}$ & .543 & 1.27 & $\begin{array}{l}0.91, \\
1.79)\end{array}$ & .163 & 1.57 & $\begin{array}{l}(1.03, \\
2.40)\end{array}$ & $.038 \underline{b}$ \\
\hline \multicolumn{13}{|l|}{ Gender } \\
\hline Female & 1.34 & $\begin{array}{l}(1.17, \\
1.54) \\
\end{array}$ & $<.001 \underline{b}$ & 1.18 & $\begin{array}{l}1.05, \\
1.34) \\
\end{array}$ & $.008 \underline{b}$ & 1.29 & $\begin{array}{l}1.09, \\
1.53) \\
\end{array}$ & $.003 \underline{b}$ & 1.17 & $\begin{array}{l}1.07, \\
1.28) \\
\end{array}$ & $<.001 \underline{b}$ \\
\hline Male & 1 & - & - & 1 & - & - & 1 & - & - & 1 & - & - \\
\hline Race/Ethnicity & & & $<.001 \underline{b}$ & & & $<.001 \underline{b}$ & & & $<.001 \underline{b}$ & & & $<.001 \underline{b}$ \\
\hline Mexican American & 0.50 & $\begin{array}{l}(0.39, \\
0.64)\end{array}$ & $<.001 \underline{b}$ & 0.51 & $\begin{array}{l}(0.37, \\
0.71) \\
\end{array}$ & $<.001 \underline{b}$ & 0.51 & $\begin{array}{l}(0.34, \\
0.77) \\
\end{array}$ & $.001 \underline{b}$ & 0.42 & $\begin{array}{l}(0.34, \\
0.51)\end{array}$ & $<.001 \underline{b}$ \\
\hline Non-Hispanic Black & 0.58 & $\begin{array}{l}0.46, \\
0.74) \\
\end{array}$ & $<.001 \underline{b}$ & 0.77 & $\begin{array}{l}0.63, \\
0.92) \\
\end{array}$ & $.005 \underline{b}$ & 0.61 & $\begin{array}{l}0.48, \\
0.76) \\
\end{array}$ & $<.001 \underline{b}$ & 0.79 & $\begin{array}{l}0.65, \\
0.97) \\
\end{array}$ & $.022 \underline{b}$ \\
\hline Non-Hispanic White & 1 & - & - & 1 & - & - & 1 & - & - & 1 & - & - \\
\hline Others & 0.55 & $\begin{array}{l}(0.44, \\
0.69)\end{array}$ & $<.001 \underline{b}$ & 0.56 & $\begin{array}{l}(0.45, \\
0.70)\end{array}$ & $<.001 \underline{b}$ & 0.60 & $\begin{array}{l}(0.48, \\
0.74)\end{array}$ & $<.001 \underline{b}$ & 0.71 & $\begin{array}{l}\text { (0.60, } \\
0.83)\end{array}$ & $<.001 \underline{b}$ \\
\hline Education level & & & $<.001 \underline{\mathrm{b}}$ & & & $<.001 \underline{b}$ & & & $<.001 \underline{b}$ & & & $<.001 \underline{b}$ \\
\hline$\leq$ Grade $12 /$ Grade 12 with no diploma & 0.69 & $\begin{array}{l}(0.56, \\
0.86)\end{array}$ & $.001 \underline{b}$ & 0.81 & $\begin{array}{l}(0.68, \\
0.97)\end{array}$ & $.022 \underline{b}$ & 0.57 & $\begin{array}{l}(0.38, \\
0.86)\end{array}$ & $.008 \underline{b}$ & 0.63 & $\begin{array}{l}(0.47, \\
0.85)\end{array}$ & $.003 \underline{b}$ \\
\hline High School graduate/GED/equivalent & 0.78 & $\begin{array}{l}(0.62, \\
1.00)\end{array}$ & $.046 \underline{b}$ & 0.77 & $\begin{array}{l}\text { (0.59, } \\
1.01) \\
\end{array}$ & .058 & 0.92 & $\begin{array}{l}(0.69, \\
1.23) \\
\end{array}$ & .557 & 0.69 & $\begin{array}{l}(0.53, \\
0.90)\end{array}$ & $.007 \underline{b}$ \\
\hline Some college or associate degree & 1 & - & - & 1 & - & - & 1 & - & - & 1 & - & - \\
\hline College graduate or above & 1.44 & $\begin{array}{l}(1.16, \\
1.79)\end{array}$ & $.001 \underline{b}$ & 1.74 & $\begin{array}{l}(1.37, \\
2.20)\end{array}$ & $<.001 \underline{b}$ & 2.18 & $\begin{array}{l}(1.73, \\
2.74)\end{array}$ & $<.001 \underline{b}$ & 1.63 & $\begin{array}{l}(1.37, \\
1.93)\end{array}$ & $<.001 \underline{b}$ \\
\hline Poverty income ratio & & & $<.001 \mathrm{~b}$ & & & $<.001 \mathrm{~b}$ & & & $<.001 \mathrm{~b}$ & & & $<.001 \mathrm{~b}$ \\
\hline
\end{tabular}




\begin{tabular}{|c|c|c|c|c|c|c|c|c|c|c|c|c|}
\hline$<100 \%$ & 0.45 & $\begin{array}{l}(0.36, \\
0.56)\end{array}$ & $<.001 \underline{\mathrm{b}}$ & 0.44 & $\begin{array}{l}(0.33, \\
0.58)\end{array}$ & $<.001 \underline{b}$ & 0.45 & $\begin{array}{l}(0.35, \\
0.58)\end{array}$ & $<.001 \underline{b}$ & 0.48 & $\begin{array}{l}(0.38, \\
0.60)\end{array}$ & $<.001 \underline{b}$ \\
\hline $100 \%-199 \%$ & 0.51 & $\begin{array}{l}\text { (0.42, } \\
0.62)\end{array}$ & $<.001 \underline{\mathrm{b}}$ & 0.47 & $\begin{array}{l}0.35, \\
0.63)\end{array}$ & $<.001 \underline{b}$ & 0.45 & $\begin{array}{l}(0.37, \\
0.55)\end{array}$ & $<.001 \underline{b}$ & 0.46 & $\begin{array}{l}\text { (0.34, } \\
0.62)\end{array}$ & $<.001 \underline{b}$ \\
\hline $200 \%-299 \%$ & 0.61 & $\begin{array}{l}\text { (0.48, } \\
0.78)\end{array}$ & $<.001 \underline{b}$ & 0.57 & $\begin{array}{l}\text { (0.46, } \\
0.70)\end{array}$ & $<.001 \underline{b}$ & 0.53 & $\begin{array}{l}(0.42, \\
0.66)\end{array}$ & $<.001 \underline{b}$ & 0.75 & $\begin{array}{l}(0.62, \\
0.90)\end{array}$ & $.002 \underline{b}$ \\
\hline $300 \%-399 \%$ & 0.83 & $\begin{array}{l}\text { (0.65, } \\
1.05)\end{array}$ & .117 & 0.68 & $\begin{array}{l}\text { (0.51, } \\
0.90)\end{array}$ & $.007 \underline{b}$ & 0.71 & $\begin{array}{l}\text { (0.51, } \\
0.99)\end{array}$ & $.043 \underline{b}$ & 0.78 & $\begin{array}{l}\text { (0.64, } \\
0.94)\end{array}$ & $.011 \underline{b}$ \\
\hline$\geq 400 \%$ & 1 & - & - & 1 & - & - & 1 & - & - & 1 & - & - \\
\hline
\end{tabular}

$\mathrm{OR}$, odds ratio; $\mathrm{Cl}$, confidence interval.

a In survey period 2009-2010, people aged 20-29 did not answer self-reported oral health.

b Significant, P-value $<.05$. 
In general, Mexican-Americans (OR range: 0.38-0.62, all $\mathrm{P}<.001$ ) and non-Hispanic Black Americans (OR range: $0.58-0.89$ ) had lower odds of reporting "excellent or very good" oral health compared to non-Hispanic Whites. People with lower education levels had lower probabilities of reporting their oral health as "excellent or very good," but this was not consistent over time. People who were college graduates or who had higher educational levels had significantly higher and varied odds of reporting "excellent or very good" oral health (OR range: 1.40-2.25, all $\mathrm{P}<.05$ ) over the years, while those with $\leq$ Grade 12/Grade 12 with no diploma had significantly lower odds (OR range: 0.57-0.84). However, high school graduates/GED holders or those with equivalent educational levels sometimes had significantly lower odds (OR range: 0.65-0.92), when compared with those with some college/AA degree. People from relatively lower family income (PIR) ratios had significantly lower probabilities of reporting "excellent or very good" oral health. For example, people having lower than 100\% PIR had significantly lower odds of reporting "excellent or very good" oral health than those having more than or equal to $400 \%$ PIR (OR range: $0.44-0.51$, all $\mathrm{P}<.001$ ).

Results from the pooled surveys of 1999-2014 and the survey-weighted multivariable binary logistic regression were consistent with results in the main effect model, and the variables were all significant with a probability of SROH of excellent or very good (all $P<.001$ ). When considering each interaction of the 5 categorical sociodemographic factors with survey periods controlling for the main effects, period $\times$ age, period $\times$ race/ethnicity, period $\times$ PIR and period $\times$ education level were individually significant (all $P<.05)$. Using forward selection for the interaction effects, 2 significant interaction effects were added (period $\times$ age: $P=.009$ and period $\times$ education level: $P=.008$ ) while the main effects were kept significant (all $\mathrm{P}<.001$ ) in the final model (Table 3 ). Predicted probabilities for the main effects are listed in Table 3, while those with interaction effects were plotted in Figures 2 and 3. This is because there were several terms included in the interaction effects, making it difficult to interpret in a table format.

Table 3. Pooled survey-weighted multivariable analyses for self-reported oral health (excellent or very good) of US adults aged 20+ years in 1999-2014

\begin{tabular}{|c|c|c|c|c|}
\hline Surveys & 1999-2014 & & & \\
\hline Variables & OR & $(95 \% \mathrm{Cl})$ & Predicted probabilitya & $P$-value \\
\hline \multicolumn{5}{|l|}{ Gender } \\
\hline Female & 1.30 & $(1.23,1.37)$ & 0.50 & $<.001 \underline{c}$ \\
\hline Male & 1 & - & 0.43 & \\
\hline Race/Ethnicity & & & & $<.001 \underline{\mathrm{c}}$ \\
\hline Mexican American & 0.49 & $(0.44,0.54)$ & 0.27 & $<.001 \underline{c}$ \\
\hline Non-Hispanic Black & 0.68 & $(0.63,0.74)$ & 0.34 & $<.001 \underline{c}$ \\
\hline Non-Hispanic White & 1 & - & 0.43 & \\
\hline Others & 0.63 & $(0.58,0.70)$ & 0.33 & $<.001 \underline{c}$ \\
\hline Poverty income ratio & & & & $<.001 \underline{\mathrm{c}}$ \\
\hline$<100 \%$ & 0.46 & $(0.42,0.51)$ & 0.26 & $<.001 \underline{c}$ \\
\hline $100 \%-199 \%$ & 0.50 & $(0.46,0.55)$ & 0.28 & $<.001 \underline{\mathrm{c}}$ \\
\hline $200 \%-299 \%$ & 0.59 & $(0.55,0.64)$ & 0.31 & $<.001 \underline{c}$ \\
\hline $300 \%-399 \%$ & 0.73 & $(0.66,0.81)$ & 0.36 & $<.001 \underline{\mathrm{c}}$ \\
\hline$\geq 400 \%$ & 1 & - & 0.43 & \\
\hline Ageb $\underline{b}$ & & & & $<.001 \underline{\mathrm{c}}$ \\
\hline Education level $\underline{b}$ & & & & $<.001 \underline{c}$ \\
\hline Period $\underline{b}$ & & & & $<.001 \underline{c}$ \\
\hline Period $\times$ ageb & & & & $.009 c$ \\
\hline
\end{tabular}


Period $\times$ education level $\underline{b}$

$.008 \underline{c}$

OR, odds ratio; $\mathrm{Cl}$, confidence interval.

a Predicted probability of reporting "excellent or very good" self-reported oral health status estimated accounting for other variables as reference category (age: 30-39; education level: some college or associate degree; period: 2013-2014)

b Note that $\mathrm{OR}$, the corresponding $95 \% \mathrm{Cl}$ and predicted probabilities of age, education level, period and their interactions were not shown in this table, but the related predicted probabilities were presented in Figures 2 and 3.

c Significant, P-value $<.05$.

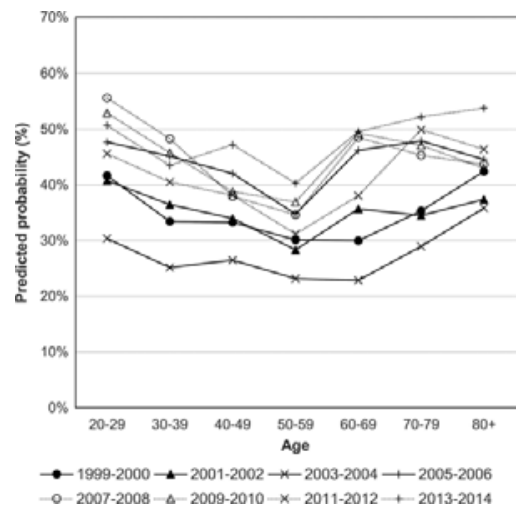

Figure 2 Predicted probability of reporting "excellent or very good" self-reported oral health status for the White males with some college or associate degree and poverty income ratio $\geq 400 \%$ in $1999-2014$, in relation to age and survey period

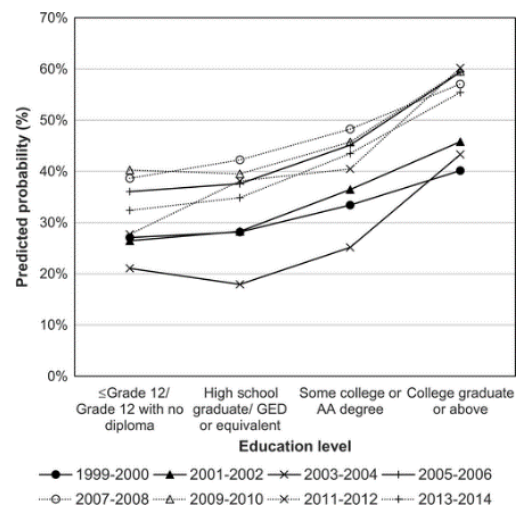

Figure 3 Predicted probability of reporting "excellent or very good" self-reported oral health status for the White males aged 30-39 y with poverty income ratio $\geq 400 \%$ in $1999-2014$, in relation to education level and survey period

In the model of pooled surveys, gender, race/ethnicity and family income (PIR) had similar and consistent results compared with those for the separate survey models mentioned above. In general, respondents in the more recent survey periods (2005-2014) had higher predicted probabilities of reporting "excellent or very good" oral health (Figures 2 and 3). Significant interaction effects between age and survey period indicated that the age effect varied with the time of the survey (Figure 2). Compared to respondents aged 30-39 years, those aged 50-59 years had relatively lower probabilities of reporting "excellent or very good" oral health while those aged 20-29 years had higher probability of reporting "excellent or very good" oral health. However, differences among other age groups varied and were not consistent over the period. 
The predicted probabilities in Figure 2 suggests that the differences among age groups were generally more or less the same from 1999 to 2006. The difference increased in 2007-2008 but then decreased slightly in 2009. The difference in 2013-2014 was almost same as that in 1999-2000. Educational level also had significant interaction effects with survey time period in the final model (Figure 3). Overall, people with a higher level of education had higher probabilities of self-reporting "excellent or very good" oral health. However, the difference between those with $\leq$ Grade 12/Grade 12 with no diploma and those who were high school graduates or had GED or equivalent education levels was not consistent over the years. The predicted probabilities in Figure 3 suggest that the difference among the educational levels generally increased from 1999 to 2004 and then stabilized with the exception of a big difference seen in 2011-2012. The goodness-of-fit for the above separated logistic models for each survey period and the logistic model for pooled survey periods was evaluated using likelihood ratio test, Score test and Wald test, and all tests demonstrated that the models were a good-fit.

\section{DISCUSSION}

In this study, we found that self-reported oral health improved from 1999 to 2014. At the same time, respondents who were young, female, had a high level of education, high income and were White reported that they had excellent/very good oral health in more recent year surveys. For investigators to detect possible time-changing effects across the surveys, interactions among the 5 sociodemographic factors in the survey period were tested while adjusting for multiple factors simultaneously. There were significant interaction terms, which implied that inequality in the sociodemographic factors changed over time while controlling for other factors. According to Peterson, 4 theoretical explanations have been proposed for inequalities in health based on Black's report published in the United Kingdom. ${ }^{33,} 34$ The 4 explanations are materialist explanation, cultural/behavioural explanation, life course perspective and the psychosocial perspective. ${ }^{33}$ Although our study is not focused directly on a particular theoretical explanation, it does provides important clues to the life course perspective that combines material, behavioural and psychosocial factors in understanding causes of social inequalities in oral health.

We found that a significant proportion of respondents self-rated their oral health in the excellent or very good categories between 1999-2000 and 2013-2014 in this study. Although not directly examined in this study, this finding may in part be related to the National Health and Nutrition Examination Survey (NHANES) results of 1988-1994, 1999-2004 and 2011-2012. These results indicate a decrease in untreated tooth decay between 1988-1994 and 1999-2004, ${ }^{35}$ and then relatively stable untreated tooth decay numbers in 2011-2012 for dentate adults 20-64 years old. ${ }^{36}$ At the same time, the excellent rating in self-reported oral health seen in the later years of the study may be associated with perceived improvements in oral health status, increased access to dental care and reduced dental disease burden in the population. Our interpretation of these findings is somewhat consistent with documented evidence that the number of missing and decayed teeth is a strong predictor of self-rated oral health in adults. ${ }^{37}$ Also, it was interesting to note that the percentage of the population reporting fair or poor oral health did not change much in this study.

Notably, there were strong associations between self-rated oral health and some demographic characteristics. There was a significant association between age and self-reported oral health. The age effect is consistent with findings from one study, ${ }^{38}$ but our results were most pronounced among the 
younger age group which showed significantly higher odds of reporting excellent and very good oral health compared to older adults. In addition, our finding that females generally rated their oral health better than males was not surprising given that being female is associated with higher rates of dental office visits, receipt of preventive care and less untreated dental disease. ${ }^{39}$ Also, our results are consistent with those published by Reisine and Bailit. ${ }^{37}$ In addition, we found a strong association between self-rated oral health and socioeconomic status characteristics. This finding is consistent with previous studies 22,40 and expands the literature related to the association between socioeconomic status and self-reported oral health. Therefore, it is important to unravel this complex relationship by engaging in studies that attempt to investigate underlying mechanism of action. Furthermore, this study did not examine possible pathways by which socioeconomic factors influence self-reported oral health. However, our finding on the association between socioeconomic factors and self-reported oral health among the poor is a potential call to action on universal coverage. According to Adler and colleague, socioeconomic status is a major determinant of health and is associated with health care, environmental exposure and health behaviour. ${ }^{41}$

Another important finding was the association between self-reported oral health and race/ethnicity. Compared to Non-Hispanic Whites, Mexican-Americans and Blacks rated their oral health negatively and had lower odds of reporting "excellent or very good" oral health. This result could be a reflection and recognition of individuals' oral health status influenced by knowledge of the healthcare system, use of services, social and cultural norms. ${ }^{42}$ In addition, Aday and Forthofer reported that MexicanAmericans were more likely to report that their oral health was poor and less likely to visit the dentist regardless of income and educational status. ${ }^{43}$ In addition, we found that education had significant interaction effects with the survey time period. This was a confirmation of the relationship between education and excellent self-rated oral health. Compared to respondents with some college or associated degree, respondents with $\leq$ Grade 12/Grade 12 with no diploma or high school/GED or equivalent education in general had a significantly lower probability of reporting excellent or very good oral health, a result consistent with previous studies that have examined this relationship. ${ }^{44,45}$ This finding is also consistent with the fact that education is key to oral health disparities, and so policies that encourage more years in school or a higher level of education will have long-term oral health benefits for individuals and population groups.

Some of the strengths of this study include our use of a nationally representative sample of the US adult population from a 15-year data set and the potential to provide information on trends of populations' SROH and effects of sociodemographic factors in predicting changes. This study has the potential to predict how well oral health programmes are doing and to inform decision makers on future programme development and planning. This study provides important information for developing and monitoring national oral health objectives. It also has the potential to aid policymakers' decisions in formulating healthy people objectives in relation to self-reported oral health measures. In addition, it provides baseline data on self-reported oral health of young and older adults in the United States. Most importantly, our study provides an opportunity for the assessment of individuals perceived oral health and population health status.

Notwithstanding, some limitations should be considered when interpreting our findings. First, this study relied on self-reported data, which could be influenced by individual and population-related factors including contextual characteristics. Second, the data are from a cross-sectional study which provides information about one time-point. Future studies should consider using data from 
longitudinal designs of adults and older adults to capture self-rated oral health over a life course. Given the fact that our data are based on a cross-sectional design, it is important to note that the interaction effect between age and survey period in the model might include the cohort effect. The cohort effect could not be easily separated because it is difficult to define mutually exclusive birth cohort categories, and this increases the complexity of interpreting cohort effect due to the survey period categories (2year cycles) in NHANES. In addition, the possible birth cohort clustering effects among the repeated cross-sectional surveys were not considered in this study. ${ }^{46}$ Furthermore, if a multilevel model was used to consider the possible birth cohort clustering effect, it would not be sufficient to generate the pseudo-likelihood estimation of weights at each level considering the design weights in complex survey designs. Therefore, this study assumed the birth cohort clustering effect to be small, and it was not considered in the analyses.

We recognize that inequality can be described in terms of absolute (in terms of rate difference) or relative (in terms of rate ratio) inequalities. Cunha-Cruz's et al ${ }^{25}$ reported on the absolute prevalence difference in edentulism between low and high socioeconomic positions that remained unchanged between 1972 and 2001 in the United States. Celeste's et al ${ }^{26}$ reported on trends in absolute prevalence difference in edentulism between lower and higher income groups in Sweden (1968-2000) and Brazil (1986-2002) that decreased, while the relative disparities remained basically the same. On the other hand, Bernabe and Sheiham ${ }^{28}$ reported that the absolute inequalities in tooth loss narrowed while relative inequalities increased steadily in the United Kingdom in 1988-2009. Trends in absolute and relative inequalities may differ, and thus, there is still debate as to whether absolute or relative inequality should be the focus in reducing inequality. ${ }^{47,48}$ In addition, methods such as Slope Index of Inequality (SII) and the Relative Index of Inequality (RII) have also been used to measure absolute and relative inequalities. ${ }^{28,49}$ However, these analytical methods were not performed in this study, given the robustness of our analytical approach to address our specific research question. In conclusion, people who are young, females, White, with high levels of education or high income reported better oral health. Future studies should assess the mechanism that underpins the association of selfreported oral health and the significant covariates and the trends in inequality to help elucidate a discussion on how our findings can be used to develop multicultural oral health programme.

\section{REFERENCES}

1Locker D. Issues in measuring change in self-reported perceived oral health status. Community Dent Oral Epidemiol. 1998; 26: 41- 47.

2Locker D. Measuring oral health: a conceptual framework. Community Dent Health. 1988; 5: 3- 18.

3 Guyatt GH, Bombardier C, Tugwell PX. Measuring disease-specific quality of life in clinical trials. CMAJ. 1986; 134: 889- 895.

4Locker D, Wexler E, Jokovic A. What do older adults' global self-ratings of oral health measure? J Public Health Dent. 2005; 65: 146- 152.

5Guyatt G, Walter S, Norman G. Measuring change over time: assessing the usefulness of evaluative instruments. J Chron Dis. 1987; 40: 171- 178.

6Dolan TA, Gooch BF, Bourque LB. Associations of self-reported dental health and general health measures in the Rand Health Insurance Experiment. Community Dent Oral Epidemiol. 1991; 19: 1- 8.

7Shen J, Wildman J, Steele J. Measuring and decomposing oral health inequalities in an UK population. Community Dent Oral Epidemiol. 2013; 41: 481- 489. 
8Locker D. Applications of self-reported assessments of oral health outcomes. J Dent Educ. 1996; 60: 494- 500.

9Thomson WM, Mejia GC, Broadbent JM, Poulton R. Construct validity of Locker's global oral health item. J Dent Res. 2012; 91: 1038- 1042.

10Kaplan G, Baron-Epel O. What lies behind the subjective evaluation of health status? Soc Sci Med. 2003; 56: 1669- 1676.

11LaRue A, Bank L, Jarvik L, Hetland M. Health in old age: how do physicians' rating and self-rating compare? J Genrotology. 1979; 34: 687- 691.

12Jokovic A, Locker D, Guyatt G. What do children's global ratings of oral health and well-being measure? Community Dent Oral Epidemiol.2005; 33: 205- 211.

13Allen PF. Assessment of oral health related quality of life. Health Qual Life Outcomes. 2003; 1: 40.

14Slade GD. Derivation and validation of a short-form oral health impact profile. Community Dent Oral Epidemiol. 1997; 25: 284- 290.

15Slade GD, Spencer AJ. Development and evaluation of the oral health impact profile. Community Dent Oral Epidemiol. 1994; 11: 3- 11.

16John MT, Koepsell TD, Hujoel P, Miglioretti DL, LeResche L, Micheelis W. Demographic factors, denture status and oral health-related quality of life. Community Dent Oral Epidemiol. 2004; 32: 125- 132.

17Pattussi MP, Peres KG, Boing AF, Peres MA, da Costa JS. Self-rated oral health and associated factors in Brazilian elders. Community Dent Oral Epidemiol. 2010; 38: 348- 359.

18Kim HY, Patton LL. Intra-category determinants of global self-rating of oral health among the elderly. Community Dent Oral Epidemiol. 2010; 38: 68- 76.

19Locker D. Self-esteem and socioeconomic disparities in self-perceived oral health. $J$ Public Health Dent. 2009; 69: 1- 8.

20Matthias RE, Atchison KA, Lubben JE, De Jong F, Schweitzer SO.Factors affecting self-ratings of oral health. J Public Health Dent. 1995; 55: 197- 204.

21Paterson JE, Gao W, Sundborn G, Cartwright S. Maternal self-report of oral health in six-year-old Pacific children from South Auckland, New Zealand. Community Dent Oral Epidemiol. 2011; 39: 19- 28.

22Atchison KA, Gift HC. Perceived oral health in a diverse sample. Adv Dent Res. 1997; 11: $272-280$.

23Dolan TA, Peek CW, Stuck AE, Beck JC. Three-year changes in global oral health rating by elderly dentate adults. Community Dent Oral Epidemiol. 1998; 26: 62- 69.

24Schuller AA. Better oral health, more inequality-empirical analysis among young adults. Community Dent Health. 1999; 16: 154- 159.

25Cunha-Cruz J, Hujoel PP, Nadanovsky P. Secular trends in socio-economic disparities in edentulism: USA, 1972-2001. J Dent Res. 2007; 86: 131- 136.

26Celeste RK, Nadanovsky P, Fritzell J. Trends in socioeconomic disparities in oral health in Brazil and Sweden. Community Dent Oral Epidemiol. 2011; 39: 204- 212.

27Holst D, Schuller AA. Equality in adults' oral health in Norway. Cohort and cross-sectional results over 33 years. Community Dent Oral Epidemiol. 2011; 39: 488- 497.

28Bernabé E, Sheiham A. Tooth loss in the United Kingdom-trends in social inequalities: an age-periodand-cohort analysis. PLOS ONE.2014; 9: e104808.

29 US Department of Health and Human Services, National Center for Health Statistics, Centers for Disease Control and Prevention.Questionnaires, datasets, and related documentation. National Health and Nutrition Examination 
Survey; 2015. http://www.cdc.gov/nchs/nhanes/nhanes questionnaires.htm. Accessed October 31, 2016.

30Johnson CL, Dohrmann SM, Burt VL, Mohadjer LK. National Health and Nutrition Examination Survey: sample design, 2011-2014. Vital Health Stat 2. 2014; 162: 1- 33.

31 US Department of Health \& Human Services, Office of the Assistant Secretary for Planning and Evaluation. Frequently asked questions related to the poverty guidelines and poverty. https://aspe.hhs.gov/frequently-asked-questions-related-poverty-guidelines-andpoverty. Accessed October 31, 2016.

32Mirel LB, Mohadjer LK, Dohrmann SM, et al. National Health and Nutrition Examination Survey: estimation procedures, 2007-2010. Vital Health Stat. 2013; 2: 1- 17.

33Sisson KL. Theoretical explanations for social inequalities in oral health. Community Dent Oral Epidemiol. 2007; 35: 81- 88.

34Peterson P. Social inequalities in dental health: towards and theoretical explanation. Community Dent Oral Epidemiol. 1990; 18: 153- 158.

35Dye BA, Tan S, Smith V, et al. Trends in oral health status: United States, 1988-1994 and 19992004. Vital Health Stat 11. 2007; 248: 1- 92.

36Dye BA, Thornton-Evans G, Li X, lafolla TJ. Dental caries and tooth loss in adults in the United States, 2011-2012. NCHS Data Brief. 2015; 197: 197.

37Reisine ST, Bailit HL. Clinical oral health status and adult perceptions of oral health. Soc Sci Med Med Psychol Med Sociol. 1980; 14A: 597- 605.

38Liu H, Maida CA, Spolsky VW, et al. Calibration of self-reported oral health to clinically determined standards. Community Dent Oral Epidemiol. 2010; 38: 527- 539.

39 US Department of Health and Human Services. Oral Health in America: A Report of the Surgeon General. Rockville, MD: US Department of Health and Human Services, National Institute of Dental and Craniofacial Research, National Institutes of Health;2000.

40Ries P. Health of black and white Americans, 1985-87. Vital Health Stat 10. 1990; 171: 1- 114.

41Adler NE, Newman K. Socioeconomic disparities in health: pathways and policies. Health Aff. 2002; 21: 60- 76.

42Aday LA, Andersen R. Development of Indices of Access to Medical Care. Ann Arbor: Health Administration Press; 1975.

43Aday LA, Forthofer RN. A profile of black and Hispanic subgroups' access to dental care: findings from the National Health Interview Survey. J Public Health Dent. 1992; 52: 210- 215.

44Bernabé E, Watt RG, Sheiham A, et al. The influence of sense of coherence on the relationship between childhood socioeconomic status and adult oral health-related behaviours. Community Dent Oral Epidemiol. 2009; 37: 357- 365.

45Lintula T, Laitala V, Pesonen P, et al. Self-reported oral health and associated factors in the North Finland 1966 birth cohort at the age of 31. BMC Oral Health. 2014; 14: 155.

46Robinson WR, Utz RL, Keyes KM, Martin CL, Yang Y. Birth cohort effects on abdominal obesity in the United States: the Silent Generation, Baby Boomers and Generation X. Int J Obes (Lond). 2013; 37: 1129- 1134.

47Mackenbach JP, Martikainen P, Menvielle G, de Gelder R. The arithmetic of reducing relative and absolute inequalities in health: a theoretical analysis illustrated with European mortality data. $J$ Epidemiol Community Health. 2016; 70: 730- 736.

48Mackenbach JP. Should we aim to reduce relative or absolute inequalities in mortality? Eur J Public Health. 2015; 25: 185. 
49Capurro DA, lafolla T, Kingman A, Chattopadhyay A, Garcia I.Trends in income-related inequality in untreated caries among children in the United States: findings from NHANES I, NHANES III, and NHANES 1999-2004. Community Dent Oral Epidemiol. 2015; 43: 500- 510. 